\title{
Physiological and pathological factors affecting the homeostasis of iron
}

\author{
Jacek H. Zeckei, Anna K. Obuchowicz \\ Clinical Department of Paediatrics, School of Health Sciences in Katowice, Medical University of Silesia, Bytom, Poland
}

\section{ABSTRACT}

Iron plays a key role in many physiological body life processes, including determination of the correct erythropoiesis. Its lack leads to the development of anaemia, and excess iron can be toxic to the body. Iron homeostasis is supervised by the interaction of many proteins, of which hepcidin plays a very important role and whose modulators are transferrin, ferroportin, and erythropoietin. Important factors modifying iron homeostasis include hypoxia, anaemia, and vitamin concentration as well as acute and chronic inflammatory processes. Hepcidin as an acute phase protein can also be an important element in the diagnosis of infectious chronic diseases, especially as an anaemic determinant of these diseases. Its role in the pathomechanism of acute inflammatory states is not entirely clear.

\section{KEY WORDS:}

iron homeostasis, hepcidin, haemojuvelin, transferrin, erythropoietin, hypoxia, anaemia.

\section{IRON HOMEOSTASIS IN THE HUMAN ORGANISM}

The total iron content of the human organism is estimated at approximately $4 \mathrm{~g}$, with $2 / 3$ of that amount being incorporated in haemoglobin in the process of erythropoiesis and the remaining part being stored in hepatocytes and cells of the reticuloendothelial system, and bound to specific proteins - ferritin, hemosiderin, and myoglobin in muscles $[1,2]$.

Daily iron demand is approximately $30 \mathrm{mg}$ and is associated with processes of production of red blood cells (RBCs) and synthesis of haemoglobin. An essential role in these processes is played by the release of iron from erythroblasts decomposing in the reticuloendothelial system, and transport of the element to erythroblasts, supervised by transferrin (Tf). At the same time, the body loses iron daily as a result of exfoliation of the epidermis, destruction of erythrocytes, and during menstrual bleeding.
The systemic processes that ensure the balance between iron loss and iron absorption from food occurring in the initial part of the duodenum are supervised by a set of proteins. They also guarantee the use of iron in the bone marrow and its recovery from phagocytised RBCs $[1,2]$.

In a properly balanced diet, about $10-20 \mathrm{mg}$ of iron is delivered daily, of which about $10 \%$ is absorbed in the intestine. The active absorption may be significantly increased in the case of increased demand. The intestinal absorption of iron $\mathrm{Fe} 3+$ (the main nutrient) depends on the proper functioning of the divalent metal transporter (DMT1), requiring a proper activity of the ferrireductase (duodenal cytochrome b - cybrd1), the enzyme located in the brush border of mature intestinal cells and supervising processes of reduction of nutritional iron $(\mathrm{Fe} 3+)$ to the accepted Fe2+ ion. DMT1 enables the transfer of the $\mathrm{Fe} 2+$ ion into enterocytes, where part of the iron is used by the cell or becomes stored [3]. Exfoliation of enterocytes into the intestinal lumen leads to elimination of

\section{ADDRESS FOR CORRESPONDENCE:}

Jacek H. Zeckei, Clinical Department of Paediatrics, School of Health Sciences in Katowice,

Medical University of Silesia, 15 Batorego St., 41-902 Bytom, Poland, e-mail: drjacekz@gmail.com 
iron. The other part of iron is transported by ferroportin through the basolateral membrane of enterocytes. The transport of $\mathrm{Fe} 2+$ through the basal membrane depends on the process of oxidation to $\mathrm{Fe} 3+$, supervised by hephaestin. The Fe3+ ion binds to apotransferrin, forming transferrin - the principal plasma-based transporter of iron in the organism. Ferroportin is also present in the cellular membrane of macrophages and in trophoblast cells, and it allows the transport of iron accumulated from phagocytised RBCs to the circulating pool bound with transferrin, as well as the transplacental transfer of iron $\mathrm{Fe} 2+$ to the foetus. Most of the cells in the body have a transferrin receptor, TfR-1, allowing iron uptake from the circulating pool $[3,4]$.

Iron is a widespread element, which plays a key role in many reactions and metabolic processes important for the proper functioning of the organism. The element participates in numerous enzymatic reactions and red-ox processes. Its principal role is building the heme molecule. By affecting the proper functioning of haemoproteins (haemoglobin, myoglobin, cytochromes of the respiratory chain, cytochrome $\mathrm{P} 450$, catalase), it participates in oxygen transfer processes [5]. It is also a component of non-heme proteins, such as ribonucleotide reductase and ferrochelatase, taking part in enzymatic reactions involving transfer of electrons. Due to its physical and chemical properties, and in particular due to its ability to undergo oxidation and reduction $(\mathrm{Fe} 3+, \mathrm{Fe} 2+)$, the possible use of the element by the organism is limited. An excess of iron may also be toxic to the organism, through participation in the production of free radicals that damage DNA, leading to protein destruction and lipid peroxidation. The process of tissue fibrosis is also a toxic effect of excessive iron [5-7].

The regulation of iron homeostasis depends on its absorption in the gastrointestinal tract and storage in hepatocytes and cells of the reticuloendothelial system. Ensuring the correct iron concentration is important because the deficiency of this element leads to anaemia, and its excess leads to serious primary and secondary metabolic disorders such as: haemochromatosis, thalassemia, and anaemia of chronic diseases [8]. Precise and strictly controlled iron concentration is directly related to its contribution to the development of infection. Iron easily available to microorganisms promotes their multiplication and facilitates the development of an acute phase of infection and of chronic infectious diseases. Bacteria use transporter proteins, i.e. transferrin, lactoferrin, and haemoglobin, as the principal source of iron. The response of the organism to the infection is activation of defence mechanisms by limiting access iron resources for pathogens [9]. For that purpose, processes of synthesis of iron-binding proteins, which participate in the transmembrane transport and carry iron with blood, are activated. In addition, the absorption of iron from the diet and the use of iron for erythropoiesis are reduced, the synthesis of haemoglobin is increased, and apolactoferrin is liberated from neutrophils. That regulation of the iron metabolism often leads to anaemia, especially in the case of chronic inflammatory diseases $[9,10]$.

\section{THE ROLE OF HEPCIDIN IN THE REGULATION OF IRON METABOLISM}

Recent research has shown that hepcidin plays one of the most important roles in the regulation of iron metabolism. It is a polypeptide made of 25 amino acids, synthesised mostly in hepatocytes and liberated to blood. The hepcidin-coding gene (hepcidin antimicrobial peptide - HAMP) is located in chromosome 19 (locus 19q13) and codes preprohepcidin. Under the effect of convertases and subsequent action of proteases the basic form is transformed, first to an intermediate form of prohepcidin, and finally to the active form of the peptide hormone hepcidin, present in the plasma and urine. The expression of hepcidin is also found in macrophages, adipocytes and cells of the renal tubules [11]. As a relatively small protein structure, it is quickly filtered by glomeruli, and thus the total hepcidin concentration in the urine is complementary to the intensity of its synthesis. Moreover, two forms of hepcidin (20- and 22-amino acid) are found in urine, probably resulting from the degradation of its active form and having no physiological significance $[11,12]$.

Hepcidin regulates iron transport by binding to ferroportin in membranes of macrophages, enterocytes, hepatocytes, and kidney cells, causing its degradation in lysosomes. The reduction of ferroportin activity leads to the exclusion of the cellular transport of iron, which results in iron retention in intestinal cells and reduced iron concentration in the organism, as a result of exfoliation of enterocytes into the intestinal lumen [4]. A similar mechanism is observed in macrophages, where the inactivation of ferroportin leads the cessation of iron release from phagocytised RBCs, and its retention in mast cells. Those processes lead to the reduction of the plasma iron level. On the other hand, in the case of a decreased concentration of hepcidin, the intestinal absorption of iron and the outflow of iron from macrophages are increased [3]. The factor that initiates the increase in hepcidin production is the growing iron storage. Excessive deposition of iron in the organism is prevented by inhibition of intestinal absorption of the element. Numerous proteins play an important role in induction of hepcidin synthesis. Roles of transferrin, transferrin 1 and 2 receptors (TfR 1 and TfR2), HFE protein (high Fe - human hemochromatosis protein), haemojuvelin (HJV), and bone morphogenetic protein 6 (BMP6) are the best known $[13,14]$. The interaction between HFE and TfR is crucial for the correct modulation of iron homeostasis and the proper expression of hepcidin function because it protects against the excessive accumulation of iron in the organism. BMP6 plays also a very important role in the regulation of hep- 
cidin synthesis, through autocrine and paracrine binding to receptors located on the surface of hepatocytes and by phosphorylation of signal proteins (SMAD 1/5/8 and SMAD 4) [13]. The study by Meynard et al. in the murine model demonstrated that an increase in iron concentration increased the expression of BMP6 mRNA, and the absence of the BMP6 gene led to iron overload. Haemojuvelin - a membrane protein found in skeletal muscles, hepatocytes, and the cardiac muscle - is the receptor for BMP and the factor potentiating its effect. The blood serum contains soluble forms of haemojuvelin (sHJV), which bind to the BMP6 protein, prevent its binding to receptors on cell membranes of liver cells, resulting in a decrease in hepcidin concentration. HJV release is directly regulated by the degree of transferrin saturation and is the result of increased muscle cells' demand for iron, for the synthesis of myoglobin, or in the case of hypoxia, and after physical exercise [15]. An increase in iron concentration causes a reduction in the release of haemojuvelin, which in turn leads to an increase in hepcidin concentration. The activity of HFE and TfR1 (located on the majority of cells) and TfR2 (located mainly on the surface of hepatocytes) is a separate way of regulating the production of hepcidin depending on the content in the organism [16]. HFE and iron-saturated transferrin compete for binding sites in TfR1. Different affinities of receptors to transferrin and to binding of different HFE domains by TfR 1 and TfR 2 directly influences the regulation of hepcidin synthesis in the liver. High plasma concentrations of transferrin-bound iron result in the dissociation of the HFE and TfR1 complex and HFE binding to the TfR2 receptor, the stabilisation of which is associated with the process of combining with transferrin (TfR2-Tf). The TfR2-Tf complex, combined with the released HFE proteins from TfR1 receptors, leads to increased intracellular production of hepcidin. Moreover, the HFE protein plays the role of an inhibitor of the iron absorption process in enterocytes and iron liberation from macrophages, and the process is independent of TfR2 $[5,8,16,17]$.

The increase in erythropoietin concentration increases the absorption of iron and stimulates erythropoiesis in the bone marrow, thereby reducing plasma iron concentration. This indirectly reduces the expression of hepcidin in cells. The direct effect of erythropoietin through the receptor (EPO-R) on the hepatocyte surface, with subsequent reduction of hepcidin synthesis, is still under discussion [1].

\section{THE EFFECT OF HYPOXIA, ANAEMIA, AND INFLAMMATION ON THE SYNTHESIS OF HEPCIDIN AND HOMEOSTASIS OF IRON}

Hypoxia is an important suppressing factor for the synthesis of hepcidin. Inhibition of production of the protein is a result of accumulation of the hypoxia-inducible protein HIF1 (hypoxia-inducible factor 1). The protein is translocated into the nucleus and regulates transcription of numerous genes, as well as binding to the promoter of the hepcidin gene [18]. Experiments demonstrated also that anaemia influenced the expression of the hepcidin gene. Iron-deficient diet results in decreased expression of hepcidin [19]. It is believed that the peptide plays an important role in the pathogenesis of anaemia of chronic diseases such as cancer, chronic infections, or autoimmune diseases. In their study, Dallalio et al. demonstrated that low concentrations of erythropoietin inhibited the proliferation and survival of erythropoietic cells by hepcidin, which is additional evidence of the involvement of hepcidin in the pathogenesis of anaemia of chronic diseases. The effect of hypoxia in the course of acute infections (of respiratory tract, for example) and its long-term effect on iron metabolism disorders, and in particular the correlation with iron resources of the organism, are not fully understood $[18,19]$. Considering the high incidence of acute and chronic infections, the regulation of iron metabolism in their course requires further and detailed research.

In the case of a chronic inflammation, the hepcidin level increases, and the increase is as an element of the organism's defence response, aimed at limiting the availability of iron for pathogens that trigger an inflammation. The final result, typical for chronic inflammations, is anaemia, resulting from the increased synthesis of proinflammatory cytokines, but also of hepcidin [20]. During chronic inflammation there is an increase in erythrophagocytosis and a reduction in RBC survival, because of the action of TNF- $\alpha$. IL-1 and TNF- $\alpha$ cause iron sequestration in macrophages and reduction of its absorption, which leads to restriction of the use of iron by precursor cells of the erythropoietic pathway [20,21]. Proinflammatory cytokines inhibit also proliferation of erythrocyte progenitor cells. Another effect of chronic inflammation is a reduction in the outflow of iron from macrophages and a decrease in its absorption in the gastrointestinal tract. This is the effect of reduced activity of ferroportin on the surface of cells by proteins regulating the iron turnover, as well as a result of the subsequent increase in hepcidin secretion [19-21]. Scientific studies have shown a significant increase in hepcidin concentration in patients with anaemia caused by chronic infections and acute inflammations. The results of experimental studies, however, indicate an increase in the expression of hepcidin under the influence of IL-1, IL-6, and lipopolysaccharide from cell membranes of Gram-negative bacteria [20,21]. That suggests a principal role of hepcidin as the acute-phase protein. The significance of the role of hepcidin synthesis and the cooperation of hepcidin and haemojuvelin in the acute phase of infection in the aspect of long-term regulation of iron metabolism requires further research $[10,22,23]$. 


\section{THE EFFECT OF VITAMINS ON IRON HOMEOSTASIS THROUGH THE REGULATION OF HEPCIDIN}

The results of scientific research indicate the role of vitamins in the regulation of expression of the hepcidin gene. Vitamin D3 was shown to reduce the hepcidin, ferritin, and proinflammatory cytokines (IL-1 and IL-6) levels, as well as to increase the expression of mRNA for ferroportin. It is postulated that vitamin D3 (also used in supplementation doses) may suppress the HAMP gene in diseases associated with high hepcidin levels [24, 25]. In vitro tests also showed a significant effect of vitamin $\mathrm{C}$ on the reduction of hepcidin mRNA and increased activity of erythropoietin and its receptors [26]. Vitamin A was demonstrated to be involved in the regulation of iron metabolism by reducing hepcidin expression and promoting iron deposition in hepatic cells, and this effect is similar to that caused by hypoxia and inflammatory processes $[27,28]$.

\section{RENAL DISEASES AND IRON METABOLISM}

The kidneys are among the main organs in which hepcidin is clearly expressed, and they constitute important regulatory organs of iron metabolism. The mechanism of impaired regulation of the iron metabolism is well known in chronic kidney disease [29].

In many kidney diseases, and in particular in chronic inflammatory processes, glomerular filtration decreases, which leads to accumulation of hepcidin and a rise of its level in blood serum. A common final effect is anaemia, which develops as a result of the mechanism of increased hepcidin and ferritin levels, the decrease in blood concentration of available iron, and consequently the decrease in haemoglobin level, as a result of impaired erythropoiesis $[1,30,31]$. In the course of chronic inflammation of kidneys, the production of pro-inflammatory cytokines, the activation of the HAMP gene, and the increase of hepcidin synthesis occurs. The main modulator of the hepcidin level is the degree of iron saturation of transferrin, which is significantly negatively affected by erythropoietin. Chronic inflammation of kidneys and clinically significant iron deficiency are the main causes of resistance of this group of patients to erythropoietin [32]. They both change the expression of hepcidin. Due to the increase in hepcidin and prohepcidin levels in patients with chronic kidney disease, correlating with the degree of renal failure, which is due to inflammation and impairment of glomerular filtration, these parameters may be potential markers of iron deficiency in chronic kidney disease [30, 31]. In addition, a positive correlation was found between serum hepcidin and ferritin levels and the inflammatory process in the organism (when the concentrations of both compounds are elevated) and in iron-deficient states (when their concentrations are lowered) [32]. There are no relevant clinical data on whether similar mechanisms also apply to acute inflammation of the upper urinary tract and whether the disturbances of iron metabolism frequently observed in acute infectious diseases, especially those of bacterial aetiology and of various localisations in the organism, may have long-term consequences.

\section{CONCLUSIONS}

Iron determines the proper course of many physiological processes. Its concentration changes in various acute and chronic diseases, including infectious and auto-inflammatory diseases. Hypoxia, anaemia, and the concentration of some vitamins are the main factors modifying iron concentration. They also have a significant impact on the broad range of proteins that modulate iron metabolism, such as hepcidin, haemojuvelin, transferrin, ferroportin, and erythropoietin. The kidneys are the main organs involved in the regulation of expression of these proteins. The results of previous studies clearly explain the mechanisms leading to their disorders in chronic diseases, especially during chronic kidney disease. However, urinary tract infections and others acute diseases may be important. Their role in the long-term consequences of iron homeostasis, especially the hepcidin-haemojuvelin axis and erythropoietin, is subject to further research.

\section{DISCLOSURE}

The authors declare no conflict of interest.

\section{REFERENCES}

1. Filipczyk L, Król P, Wystrychowski A. Hepcydyna - hormon wątrobowy kontrolujący homeostazę żelaza. Forum Nefrol 2010; 3: 233-242.

2. Beaumont C, Delaby C. Recycling iron in normal and pathological states. Semin Hematol 2009; 46: 328-338.

3. Andrews NC. Forging a field: the golden age of iron biology. Blood 2008; 112: 219-230.

4. Shayeghi M, Latunde-Dada GO, Oakhill J, et al. Identification of an intestinal heme transporter. Cell 2005; 122: 789-801.

5. Zhang AS, Enns CA. Molecular mechanisms of normal iron homeostasis. Hematology Am Soc Hematol Educ Program 2009: $207-$ 214.

6. Andrews NC. Disorders of iron metabolism. N Engl J Med 1999; 341: 1986-1995.

7. Kushner JP, Porter JP, Olivieri NF. Secondary iron overload. Hematology Am Soc Hematol Educ Program 2001: 47-61.

8. Goswami T, Andrews NC. Hereditary hemochromatosis protein, HFE, interaction with transferrin receptor 2 suggests a molecular mechanism for mammalian iron sensing. J Biol Chem 2006; 281 : 28494-28498.

9. Cherayil BJ. The role of iron in the immune response to bacterial infection. Immunol Res 2011; 50: 1-9.

10. Kossiva L, Gourgiotis DI, Tsentidis C, et al. Serum hepcidin and ferritin to iron ratio in evaluation of bacterial versus viral infection in children: a single-center study. Pediatr Infect Dis J 2012; 31: $795-$ 798. 
11. Malyszko J. Hepcidin assays: ironing out some details. Clin J Am Soc Nephrol 2009; 4: 1015-1016.

12. Park CH, Valore EV, Waring AJ, et al. Hepcidin, a urinary antimicrobial peptide synthesized in the liver. J Biol Chem 2001; 276: 7806-7810.

13. Andriopoulos B Jr, Corradini E, Xia Y, et al. BMP6 is a key endogenous regulator of hepcidin expression and iron metabolism. Nat Genet 2009; 41: 482-487.

14. Babitt JL, Huang FW, Wrighting FW, et al. Bone morphogenetic protein signalling by hemojuveline regulates hepcidin expression. Nat Genet 2006; 38: 531-539.

15. Przybyszewska J, Żekanowska E. The role of hepcidin and haemojuveline in the pathogenesis of iron disorders in patients with severe malnutrition. Ann Agric Environ Med 2014; 21: 336-338.

16. West AP, Bennett MJ, Sellers VM, et al. Comparison of the interactions of transferrin receptor and transferrin receptor 2 with the transferring and the hereditary hemochromatosis protein HFE. J Biol Chem 2000; 275: 38135-38138.

17. Schmidt PJ, Toran PT, Gianetti AM, et al. The transferring receptor modulates HFE-dependent regulation of hepcidin expression. Cell Metab 2008; 7: 205-214.

18. Nicolas G, Chauvet C, Viatte L, et al. The gene encoding the iron regulatory peptide hepcidin is regulated by anemia, hypoxia and inflammation. J Clin Invest 2002; 110: 1037-1044.

19. Weiss G, Goodnough LT. Anemia of chronic disease. N Engl J Med 2005; 352: 1011-1023.

20. Nemeth E, Rivera S, Gabayan V, et al. IL-6 mediates hypoferremia of inflamation by inducing the synthesis of the iron regulatory hormone hepcidin. J Clin Invest 2004; 113: 1271-1276.

21. Kemna E, Pickkers P, Nemeth E, et al. Time-course analysis of hepcidin, serum iron, and plasma cytokine levels in humans injected with LPS. Blood 2005; 106: 1864-1866.

22. Domenico I, Ward DM, Kaplan J. Hepcidin regulation: ironing out the details. J Clin Invest 2007; 117: 1755-1758.

23. Singh B, Arora S, Agrawal P, et al. Hepcidin: a novel peptide hormone regulating iron metabolism. Clin Chim Acta 2011; 412: 823-830.

24. Bacchetta J, Zaritsky JJ, Sea JL, et al. Suppression of iron-regulatory hepcidin by vitamin D. J Am Soc Nephrol 2014; 25: 564-572.

25. Zughaier SM, Alvarez JA, Sloan JH, et al. The role of vitamin D in regulating the iron-hepcidine-ferroportin axis in monocytes. J Clin Transl Endocrinol 2014; 1: 19-25.

26. Chiu PF, Ko SY, Chang CC. Vitamine C affects the expression of hepcidin and erythropoietin receptor in HepG2 cells. J Ren Nutr 2012; 22: 373-376.

27. Citelli M., Bittencourt LL., da Silva SV, et al. Vitamina A modulates the expression of genes involved in iron bioavailability. Biol Trace Elem Res 2012; 149: 64-70.

28. da Cunha MS, Siqueira EM, Trindade LS, et al. Vitamin A deficiency modulates iron metabolism via ineffective erythropoiesis. J Nutr Biochem 2014; 25: 1035-1044.

29. Tsuchiya K, Nitta K. Hepcidin is a potential regulator of iron chronic kidney disease. Ther Apher Dial 2013; 17: 1-8.

30. Taes YE, Wuyts B, Boelaert JR, et al. Prohepcidin accumulates in renal insufficiency. Clin Chem Lab Med 2004; 42: 387-389.

31. Zaritsky J, Young B, Wang H-J, et al. Hepcidin - a potential novel biomarker for iron status in chronic kidney disease. Clin J Am Soc Nephrol 2009; 4: 1051-1056.

32. Reichert CO, da Cunha J, Levy D, et al. Hepcidin: Homeostasis and diseases related to iron metabolism. Acta Haematol 2017; 137: 220-236. 